\title{
Vol. 65, No. 15
}

In the report "Notes from the Field: Respiratory Symptoms and Skin Irritation Among Hospital Workers Using a New

Disinfection Product - Pennsylvania, 2015," in the fourth paragraph, the fourth sentence should have read "Full-shift air sample results for hydrogen peroxide ranged from $<\mathbf{1 1}$ parts per billion (ppb) to $511 \mathrm{ppb}$; for acetic acid, from $<\mathbf{8 . 8} \mathbf{~ p p b}$ to $319.4 \mathbf{~ p p b}$; and for peroxyacetic acid, from $<\mathbf{2 . 2} \mathrm{ppb}$ to 48 ppb." 